\title{
Liquid Crystal Display Modes in a Nontilted Bent-Core Biaxial Smectic Liquid Crystal
}

\author{
Mamatha Nagaraj \\ Trinity College Dublin, Ireland \\ Yuri Panarin \\ Technological University Dublin, yuri.panarin@tudublin.ie \\ J. K. Vij \\ Trinity College Dublin, Ireland, jvjj@tcd.ie
}

See next page for additional authors

Follow this and additional works at: https://arrow.tudublin.ie/engscheleart2

Part of the Electrical and Computer Engineering Commons

\section{Recommended Citation}

Keith, C. et al. (2010). Liquid crystal display modes in a nontilted bent-core biaxial smectic liquid crystal. Applied Physics Letters, vol. 97, pp. 1-3. doi:10.1063/1.3513832

This Article is brought to you for free and open access by the School of Electrical and Electronic Engineering at ARROW@TU Dublin. It has been accepted for inclusion in Articles by an authorized administrator of ARROW@TU Dublin. For more information, please contact arrow.admin@tudublin.ie, aisling.coyne@tudublin.ie, gerard.connolly@tudublin.ie. 
Authors

Mamatha Nagaraj, Yuri Panarin, J. K. Vij, C. Keith, and C. Tschierske

This article is available at ARROW@TU Dublin: https://arrow.tudublin.ie/engscheleart2/165 


\title{
Liquid crystal display modes in a nontilted bent-core biaxial smectic liquid crystal
}

\author{
Mamatha Nagaraj, ${ }^{1}$ Y. P. Panarin, ${ }^{1,2}$ J. K. Vij, ${ }^{1, a)}$ C. Keith, ${ }^{3}$ and C. Tschierske ${ }^{3}$ \\ ${ }^{1}$ School of Engineering, Trinity College, University of Dublin, Dublin 2, Ireland \\ ${ }^{2}$ School of Electronic and Communication Engineering, Dublin Institute of Technology, Dublin, Ireland \\ ${ }^{3}$ Institute of Organic Chemistry, Martin-Luther-University Halle-Wittenberg, Halle D06120, Germany
}

(Received 28 September 2010; accepted 19 October 2010; published online 24 November 2010)

\begin{abstract}
Liquid crystal display (LCD) modes associated with the rotation of the secondary director in nontilted, biaxial smectic phase of an achiral bent-core compound are demonstrated. For LCDs, we find that at least four display modes are possible using $\mathrm{SmAP}_{\mathrm{A}}$ phase of the studied material, in which the minor directors in adjacent layers are aligned antiferroelectrically. The advantages of these modes include low driving field $(1-2 \mathrm{~V} / \mu \mathrm{m})$, high contrast ratio 1000:1, relatively fast switching time of $0.5 \mathrm{~ms}$ and continuous gray scale. The molecular short axis or the polar axis in a negative dielectric, biaxial material is oriented by the in-plane electric field by a combination dielectric biaxiality and polarity at low electric fields and polarity at higher fields. (C) 2010 American Institute of Physics. [doi:10.1063/1.3513832]
\end{abstract}

Liquid crystal displays (LCDs) have made great progress in their quality over the years due to a reduction in the cost of thin film transistor technology and with the development of the in-plane switching (IPS) (Refs. 1 and 2) and vertical alignment (VA) (Refs. 3 and 4) display modes. A contemporary LCD possesses sufficient contrast ratio, a reasonable wide viewing angle, acceptable speed, and a reasonable level of power consumption. Nevertheless, to compete with new, quickly developing technologies such as organic light emitting diodes, field-effect transistors, etc., it is important to continue to enhance the device performance. One of the main problems in further improvements in the LCD technology is the limit in the switching speed. The new LCD modes with sequential color division and strobing light-emitting diode (LED) backlight require at least three times faster switching speed than has yet been realized in the IPS and VA modes using conventional nematics so far. The possible solutions to overcome this disadvantage are the use of (i) dualfrequency control and (ii) ferroelectric liquid crystals in displays but these in turn suffer other major problems. The solutions proposed so far have therefore not yet been commercialized for large-scale production of displays.

Recently the use of some of the basic characteristics of the biaxial nematic phase has offered newer possibilities. Here the main idea is to switch the secondary director $\vec{m}$ (along the short molecular axis) rather than the primary director $\vec{n}$ (along the long molecular axis) (Fig. 1). The switching of the minor (or secondary) director is a factor of 10-100 times faster than the primary one. Biaxiality has also been observed in some of the orthogonal smectic phases of the achiral banana shaped molecules in absence of the field. The major interest in "banana-shaped" liquid crystalline materials has arisen due to their ability of forming a rich variety of subphases (B1-B8); some of these show fast switching due to the presence of the spontaneous polarization of the domains in these phases. Nevertheless systems using such phases have also not been commercialized due to the problems associated with alignment and in obtaining defect-free cells. $^{5}$

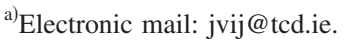

In the biaxial nematic phase of bent-core molecules, fast switching between different optical states may be possible by the rotation of the short axis $\vec{m}$ around the fixed long axis $\vec{n}$ due to the coupling of dielectric anisotropy $\left(\varepsilon_{\mathrm{m}}-\varepsilon_{1}\right)$ with the field [Figs. 1(b) and 1(c)]. However, practically it is difficult to realize an independent switching of short axis $\vec{m}$ and this is usually accompanied by unwanted tilt of the primary director $\vec{n}^{6}{ }^{6}$ Recently a number of bent-core molecular systems with orthogonal SmA-like phases have been synthesized and studied. ${ }^{7-11}$ In addition to $\mathrm{SmA}$ phase, these systems may form lower temperature $\operatorname{SmAP}_{\mathrm{R}}$ and $\mathrm{SmAP}_{\mathrm{A}}$ phases. In these orthogonal smectic phases, the molecular rotation around the major director within the smectic layer is hindered and the molecules are packed in the bent-direction or in the direction of the polar axis. In $\operatorname{SmAP}_{R}$ phase in the absence of electric field, the polar directors in different smectic layers are randomly arranged while in the $\mathrm{SmAP}_{\mathrm{A}}$ phase the polar director alternates from layer to layer forming antiferroelectric structure.

In this letter, we present results of a comparative experimental study of the different electro-optic display modes in both $\mathrm{SmAP}_{\mathrm{R}}$ and $\mathrm{SmAP}_{\mathrm{A}}$ phases of an achiral bent-core

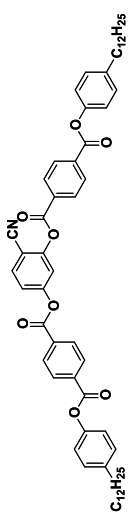

(a)

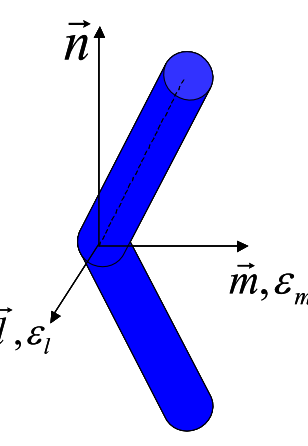

(b)

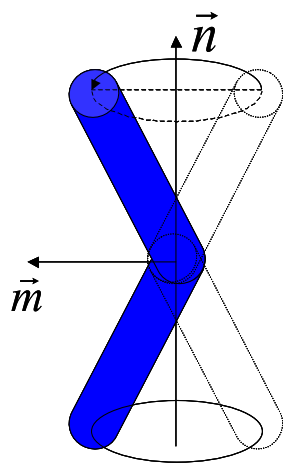

(c)
FIG. 1. (Color online) (a) Molecular structure of CK64 material ${ }^{12}$ (b) Primary $(\mathrm{n})$ and secondary $(\mathrm{m}, \mathrm{l})$ directors in a bent-core molecule, dielectric permittivities $\varepsilon_{\mathrm{m}}$ and $\varepsilon_{1}$ are along the two secondary directors "m" and "l," respectively. (c) Molecular switching around the primary director. 

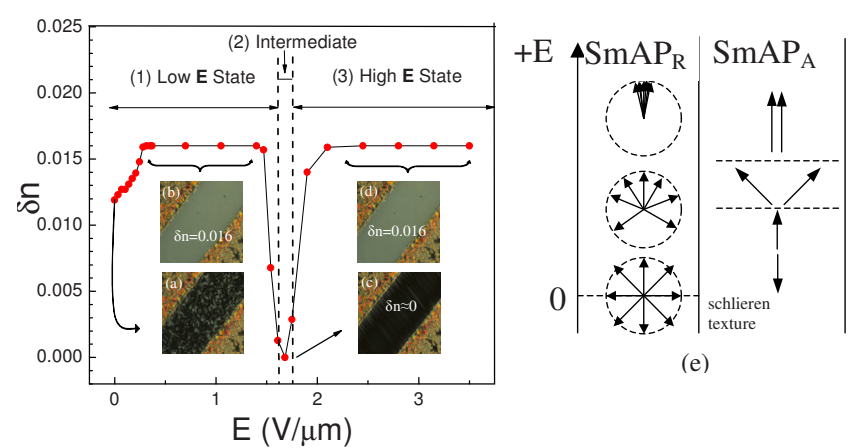

(e)

FIG. 2. (Color online) Biaxiality in $\mathrm{SmAP}_{\mathrm{A}}$ phase vs in-plane electric field (at a frequency of $110 \mathrm{~Hz}$ ) at $\mathrm{T}=110{ }^{\circ} \mathrm{C}$ in a homeotropic cell of thickness $8.7 \mu \mathrm{m}$. Insets (a), (b), (c), and (d): textures under crossed polarizers for different electric field strengths. Field direction is at an angle of $45^{\circ}$ to the polarizer/analyzer. The distance between the electrodes is $180 \mu \mathrm{m}$. (e) Switching mechanisms in $\mathrm{SmAP}_{\mathrm{R}}$ and $\mathrm{SmAP}_{\mathrm{A}}$ phases; the arrows represent the secondary molecular axes/local polarization vectors.

compound. The common principle of these modes is that of the switching of the molecular dipoles or molecular short axes by the in-plane electric field.

The compound used in our study CK64 [structure given in Fig. 1(a)] exhibits a unique sequence of phases as follows: $\mathrm{Cr} 37.8{ }^{\circ} \mathrm{C} \quad \mathrm{SmAP}_{\mathrm{A}} \quad 111{ }^{\circ} \mathrm{C} \quad \mathrm{SmAP}_{\mathrm{R}} \quad 157.5^{\circ} \mathrm{C} \quad \mathrm{SmA}$ $163.9^{\circ} \mathrm{C}$ I obtained on cooling with both $\mathrm{SmAP}_{\mathrm{A}}$ and $\mathrm{SmAP}_{\mathrm{R}}$ phases existing over a broader temperature range. ${ }^{12}$ A detailed work on electric field induced textural transformations in $\mathrm{SmAP}_{\mathrm{A}}$ phase of this sample are given elsewhere. ${ }^{13}$ The material shows negative dielectric anisotropy through out the liquid crystalline temperature range. Polymer AL60702 (JSR Korea) is used as an alignment agent for the homeotropic cells. The cell spacing is $8.7 \mu \mathrm{m}$, the distance between two stripe indium tin oxide (ITO) electrodes is $\sim 180 \mu \mathrm{m}$. On cooling, the cell with $\mathrm{LC}$ in the $\mathrm{SmAP}_{\mathrm{R}}$ phase under crossed polarizers, we find that the cell is well aligned and exhibits perfect extinction in the absence of external electric field, whereas the cell in $\mathrm{SmAP}_{\mathrm{A}}$ phase shows schlieren texture of both $\mathrm{s}= \pm 1 / 2$ and $\mathrm{s}= \pm 1$ declinations [Fig. 2 inset (a)].

For the material under investigation, we obtained one of the display modes in $\mathrm{SmAP}_{\mathrm{R}}$ phase. Shimbo et al. ${ }^{14}$ demonstrated a polarization switching process in the $\mathrm{SmAP}_{\mathrm{R}}$ phase on the application of the electric field. They proposed a LCD mode using $\mathrm{SmAP}_{\mathrm{R}}$ phase of an achiral bent-core liquid crystal from the field off to the field on state. ${ }^{15}$ The $\operatorname{SmAP}_{\mathrm{R}}$ phase is macroscopically uniaxial, since the polar directors in the plane of the smectic layers are randomly ordered in the absence of the electric field. An application of rather high electric field aligns the minor directors of this initially disordered structure and therefore induces biaxiality [Fig. 2(e)]. This display mode is advantageous over the mode in biaxial nematic phase ${ }^{6}$ due to the absence of parasitic switching of the primary director since it has been anchored in the $\operatorname{SmAP}_{R}$ phase by the layer structure itself. But the disadvantage of this switching mode is that it requires rather high electric field, which is necessary to align the initially disordered structure. Hence in order to achieve a reasonable value of the field induced optical biaxiality, electric fields of $10 \mathrm{~V} / \mu \mathrm{m}$ or higher must be applied. In homeotropic geometry this corresponds to the voltage of several hundred volts [Table I]. From this point of view the $\mathrm{SmAP}_{\mathrm{A}}$ phase may be more prospective due to the existence of the spontaneous biaxiality and its interaction with the electric field.

Figure 2 shows the dependence of biaxiality on electric field and the corresponding textures under the crossed polarizers in the $\mathrm{SmAP}_{\mathrm{A}}$ phase of a homeotropic cell under the in-plane electric field applied at a frequency of $110 \mathrm{~Hz}$. At low values of electric field $(<1.6 \mathrm{~V} / \mu \mathrm{m})$, the initial Schlieren texture transforms to a uniform biaxial texture [marked as (1), inset (b)], which with an increase in field goes to an intermediate uniaxial texture [marked as 2, inset (c)], a further increase in the field, the system goes to a ferroelectric state [marked as 3, inset (d)] with the same value of biaxiality as in the $\mathrm{SmAP}_{\mathrm{A}}$ phase. The response of $\mathrm{SmAP}_{\mathrm{A}}$ structure to the applied electric field shows three optically distinguishable states. The physical phenomena responsible for this textural transformation is presented in Fig. 2(e). Application of small electric field slightly distorts the initial antiferroelectric structure and for higher fields this antiferroelectric structure completely transforms to ferroelectric structure. In the very narrow range of intermediate $\mathrm{E}$, the homeotropic cell appears dark under crossed polarizers arising from zero biaxiality; this is possible when the angle between the local polarizations vectors (and therefore the polar directors) is $90^{\circ} .{ }^{13}$ This sequence of transition of the biaxiality is exploited in driving the display either between the low E bright state (1) to an intermediate E dark state (2) [mode 2(a)] or between the states (2) to a high E bright state (3) [mode 2(b)]. The former type of switching could be achieved at very low fields but switching time is rather large compared to the latter type of switching where we need to apply relatively moderate fields for faster switching. The electro-optic parameters of these modes are listed in Table I.

There is another possibility for obtaining bright and dark states of a display in $\mathrm{SmAP}_{\mathrm{A}}$ using a cell configuration; wherein both bottom and top glass substrates each with a set of in-plane electrodes are fixed an angle of at $45^{\circ}$ to one another. Figures 3(a) and 3(b) show such a cell configuration. When an in-plane electric field $\sim 1 \mathrm{~V} / \mu \mathrm{m}$ is applied to the first pair of electrodes (say, to the bottom electrodes), it gives a uniform texture in the active area between the electrodes since the electric field has oriented all the dipoles toward the

TABLE I. The possible LCD modes in $\mathrm{SmAP}_{\mathrm{R}}$ and $\mathrm{SmAP}_{\mathrm{A}}$ phases and their display parameters in CK64 sample.

\begin{tabular}{|c|c|c|c|c|c|c|}
\hline 1 & $\mathrm{SmAP}_{\mathrm{R}}$ & $4 \mathrm{~V} / \mu \mathrm{m}$ (near-SmAP $\mathrm{S}_{\mathrm{A}}$ transition) & 2 & $>1000: 1$ & 100 & 0.003 at $4 \mathrm{~V} / \mu \mathrm{m}$ \\
\hline 2(b) & $\mathrm{SmAP}_{\mathrm{A}}$ & $>1.7 \mathrm{~V} / \mu \mathrm{m}$ & $\sim 3$ & 200:1 & 16 & 0.016 \\
\hline $3(\mathrm{a})$ & $\mathrm{SmAP}_{\mathrm{A}}$ & $<1.5 \mathrm{~V} / \mu \mathrm{m}$ & 0.5 & $>1000: 1$ & 16 & 0.016 \\
\hline $3(\mathrm{~b})$ & $\mathrm{SmAP}_{\mathrm{A}}$ & $>1.7 \mathrm{~V} / \mu \mathrm{m}$ & $<0.4$ & $>1000: 1$ & 16 & 0.016 \\
\hline
\end{tabular}




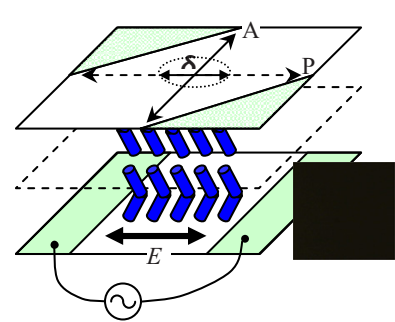

(a)

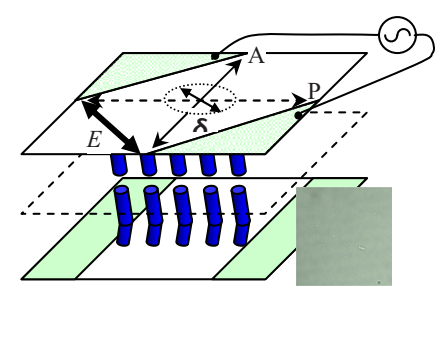

(b)
FIG. 3. (Color online) [(a) and (b)] Molecular orientations when the electric field is applied between the bottom and top pairs of electrodes, respectively, in a homeotropic cell configuration used for the IPS. Green stripes represent ITO electrodes. Inset: transmittance in the field of view for the corresponding orientations. $\mathrm{E}=1 \mathrm{~V} / \mu \mathrm{m}, 110 \mathrm{~Hz}$, and $\mathrm{T}=110^{\circ} \mathrm{C}$.

field direction. When this pair of electrodes is along the polarizer, a dark state in the field of view [Fig. 3(a) inset] is obtained. Now by sequencing the field to the second pair of electrodes, we obtain a bright region [Fig. 3(b) inset] in the field of view since the second pair of electrodes is at an angle of $45^{\circ}$ with respect to the polarizer [Fig. 3(b)]. Hence dark and bright states can be displayed under crossed polarizers at lower driving fields with lower switching times. Again, the switching can occur at lower [state (1) in Fig. 2], or higher electric fields [state (3) in Fig. 2]; these are represented as modes 3(a) and 3(b), respectively, in Table I. They are achieved due to the interaction predominantly of the dielectric biaxiality with electric field at low electric fields in mode 3(a) and polarity at higher fields, mode 3(b).

The optical transmittance of this system is expressed as follows: $T=k \sin ^{2}(2 \varphi) ; k$ depends on intensity of the source, and $\left[\sin ^{2}(\pi \Delta n d / \lambda)\right]$ is the birefringence term. $\varphi$ is the angle between the secondary director $\vec{m}$ and the polarizer axis. The contrast ratio of such bright and dark states is 1000:1; this measurement has been limited by the quality of the polarizer. Simultaneous activation of either electrodes (or set of electrodes) with different fields results in a variation in the net angle of the secondary director lying in between $0^{\circ} \leq \varphi$ $\leq 45^{\circ}$ with the polarizer; this provides a continuous gray scale. The molecular bent angle calculated using NMR (Ref. 16) provides wider viewing angles to these displays.

Figure 4 shows the measured response time (for the above cell configuration) as a function of the electric field at

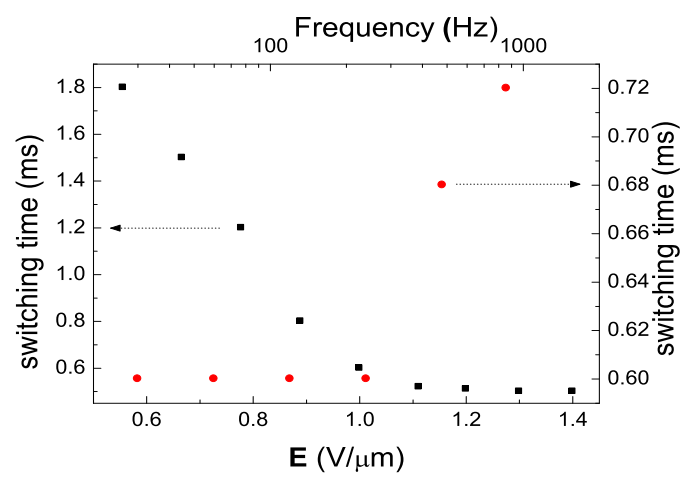

FIG. 4. (Color online) (a) Response time as a function of electric field strength at a frequency $110 \mathrm{~Hz},(\mathbf{\square})$ and as a function of frequency of $\mathrm{E}$ $=1 \mathrm{~V} / \mu \mathrm{m}(\bullet)$ at $\mathrm{T}=110^{\circ} \mathrm{C}$. a frequency of $110 \mathrm{~Hz}$ and as a function of frequency at a fixed electric field of $1 \mathrm{~V} / \mu \mathrm{m}$. The fastest response we obtain for this sample is $400 \mu$ s for fields greater than $1.7 \mathrm{~V} / \mu \mathrm{m}$. Table I lists the possible display modes in $\mathrm{SmAP}_{\mathrm{R}}$ and $\mathrm{SmAP}_{\mathrm{A}}$ phases with the display parameters obtained for CK64 material. Since the alignments of long and short axes of the molecule are fixed by the smectic phase, we do not need to use any external treatment like rubbing to obtain a uniform alignment. This provides with the advantage of high contrast ratio for the displays.

In conclusion, we demonstrate different display modes in nontilted, polar, antiferroelectric smectic phases of an achiral bent core compound with a lower threshold, high contrast, and continuous gray scale. There is a considerable advantage in realizing the switching in nontilted biaxial smectic phases since the major director is already oriented by the smectic layers unlike in biaxial nematics where it needs to be anchored independently of the secondary director by external forces which may lead to parasitic effects like induced tilt with field. It is also possible to have higher switching speed in smectics, which is suitable for sequential color display modes with LED backlight as electric field interacts with induced polarization as opposed to the dielectric biaxiality in nematics. Also since the phase is orthogonal in nature the problems associated with SmC materials (chevron structure, defects) could be avoided. Hence molecular design and synthesis of low viscosity materials having this phase at lower temperatures is essential for use in display modes.

We acknowledge funding by EU Grant No. FP7-216025 BIND and SFI Grant No. RFP 06/RFP/ENE039 projects.

${ }^{1}$ M. Oh-e and K. Kondo, Appl. Phys. Lett. 67, 3895 (1995).

${ }^{2}$ R. Kiefer, B. Weber, F. Windscheid, and G. Baur, Proceeding of 12th International Display Research Conference (Society for Information Display, Hiroshima, 1992), p. 47

${ }^{3}$ Y. Koike, S. Kataoka, T. Sasaki, H. Chida, H. Tsuda, A. Takeda, K. Ohmuro, T. Sasabayashi, and K. Okamoto, Proceeding of Fourth International Display Workshop (Society for Information Display, Nagoya, 1997), p. 159.

${ }^{4}$ G. Assouline, M. Hareng, and E. Leiba, Electron. Lett. 7, 699 (1971).

${ }^{5}$ H. Takezoe and Y. Takanishi, Jpn. J. Appl. Phys., Part 1 45, 597 (2006).

${ }^{6}$ M. Nagaraj, Y. P. Panarin, U. Manna, J. K. Vij, C. Keith, and C. Tschierske, Appl. Phys. Lett. 96, 011106 (2010).

${ }^{7}$ A. Eremin, S. Diele, G. Pelzl, H. Nádasi, W. Weissflog, J. Salfetnikova, and H. Kresse, Phys. Rev. E 64, 051707 (2001).

${ }^{8}$ M. W. Schröder, S. Diele, N. Pancenko, W. Weissflog, and G. Pelzl, J. Mater. Chem. 12, 1331 (2002).

${ }^{9}$ H. N. S. Murthy and B. K. Sadashiva, Liq. Cryst. 31, 567 (2004).

${ }^{10}$ U. Dunemann, M. W. Schroder, R. A. Reddy, and C. Tschierske, J. Mater. Chem. 15, 4051 (2005).

${ }^{11}$ D. Pociecha, E. Gorecka, M. Cepic, N. Vaupotic, and W. Weissflog, Phys. Rev. E 74, 021702 (2006).

${ }^{12}$ C. Keith, M. Prehm, Y. P. Panarin, J. K. Vij, and C. Tschierske, Chem. Commun. (Cambridge) 46, 3702 (2010).

${ }^{13}$ Y. P. Panarin, M. Nagaraj, J. K. Vij, C. Keith, and C. Tschierske, Europhys. Lett. 92, 26002 (2010).

${ }^{14}$ Y. Shimbo, E. Gorecka, D. Pociecha, F. Araoka, M. Goto, Y. Takanishi, K. Ishikawa, J. Meieczkowski, K. Gomola, and H. Takezoe, Phys. Rev. Lett. 97, 113901 (2006).

${ }^{15}$ Y. Shimbo, Y. Takanishi, K. Ishikawa, E. Gorecka, D. Pociecha, J. Mieczkowski, K. Gomola, and H. Takezoe, Jpn. J. Appl. Phys., Part 2 45, L282 (2006)

${ }^{16}$ I. Wirth, S. Diele, A. Eremin, G. Pelzl, S. Grande, L. Kovalenko, N. Pancenkoa, and W. Weissfloga, J. Mater. Chem. 11, 1642 (2001). 